\title{
Mathematical Aesthetics in a Beautiful Town: Bridges Coimbra 2011
}

Kristóf Fenyvesi

Interdisciplinary discussions on the relations between mathematics and the arts, science, aesthetics, and artistic practice have a long history. At the present time, various cultural phenomena under the influence of mathematics and the arts continue to inspire people working in different fields of science. Similarly, several artists and scholars share a common interest in combining creative thinking, intellectual curiosity, and aesthetic sensibility in their work and research. The Bridges Conferences http://www.BridgesMathArt.org, founded and lead by Reza Sarhangi (Towson University) and running annually since 1998 , aim to initiate a dialogue between the mathematical and the artistic points of view in various fields where artistic and mathematical thinking and practice merge. Unique components of the Bridges Conferences, in addition to formal presentations, are gallery displays of visual art; hands-on workshops designed for professional educators as well as for families and children; working sessions with artists and mathematicians who are crossing the mathematicsarts boundaries; mathematical poetry readings; and musical, th;atrical, and movie events in the evenings.

\section{Coimbra: The City of Sciences, Arts, and Bridges}

The 2011 Bridges Conference, codirected by Penousal Machado, was held at the University of Coimbra, Portugal, during July 27-31, 2011. The

Kristóf Fenyvesi is a researcher in the Art and Culture Studies Department at Jyväskylä University, Finland; curator-in-chief of the Ars Geometrica International Conference series, Pécs, Hungary, which hosted the Bridges World Conference in 2010; leader of the Experience Workshop Math-Art Movement; art curator of the Ars Geometrica Math-Art Gallery (Eger College, Hungary); and coordinator of community events of the Bridges Organization. His email address is fenyvesi.kristof@gmai1.com.

DOI: http://dx.doi.org/10.1090/noti846
University of Coimbra was established in 1290 as the first university in Portugal and the first Portuguese language university in the world. After the much larger metropolitan areas of Lisbon and Porto, Coimbra is the most important urban focal point of the central part of the country. It is situated on the Rio Mondego, the longest river located exclusively in Portugal. The river, which flows through the city, provides a scenic setting for the town, which invites all to walk along its river banks and across its four bridges. It was a beautiful setting for the Bridges Conference!

\section{Math Goes Fashion}

Since last year when Bridges visited Hungary, ${ }^{1}$ the conference has grown again and set new records. The conference received almost 300 paper, artwork, and proposal submissions, more than ever before, presenting intriguing ideas in mathematics, in the arts, and in several other cultural domains. This year the keynote paper was coauthored by Fields medalist William P. Thurston (Cornell University) and was presented by Kelly Delp (Buffalo State College). The spectacular presentation described a process that was inspired by modern fashion design and that can be adapted to construct many different surfaces out of paper and craft foam.

Another plenary paper was presented by Paulus Gerdes, the president of ISGEm, the International Study Group on Ethnomathematics and vice president of Southern Africa in the African Academy of Sciences. Gerdes analyzed instances of mathematical ideas interwoven in the artistic decoration of handbags, hats, mats, and other products of basketry from several regions of Mozambique. He shared instructive stories about "field encounters" with local handcrafters and touched upon such exciting phenomena as the tradition of geometrical

${ }_{1}^{1}$ Paul Gailiunas, Bridges Pécs 2010, Notices of the American Mathematical Society 58, no. 2 (February 2011), 289-290. 
imagination and the cultural role of the so-called "mental geometry" and their links to the future of mathematics education in Africa.

Among the many memorable presentations, it is also important to mention the joint paper by artist Jim R. Paulsen and Reza Sarhangi on Paulsen's impressive sculptures Sentinels. As an addition to the keynote talks, George W. Hart, a member of the Bridges board of directors and the chief of content at the Museum of Mathematics (MoMath) in New York City, offered exclusive insight for the conference participants into MoMath's most interesting innovations of interactive presentation techniques of mathematical contents in the museum.

The local organizers also realized a Portuguese math/art day, which featured invited talks by Manuel Arala Chaves (University of Porto, a member of the directing board of the Atractor ProjectInteractive Mathematics), Paulus Gerdes, and João Paulo Xavier (University of Porto), complemented by workshops on the symmetry of plane patterns, on Roman architecture in Portugal, and on Lunda art, inspired by sona geometry from Angola.

\section{Mathematics Is Art}

An exhibition of mathematical art has been an annual feature of Bridges since 2001, and it has grown steadily over the years under the dedicated leadership of Robert Fathauer. This year, work was submitted by more than seventy artists from twenty countries. Diverse artistic media were represented, including wood, metal, and stone sculptures; beadwork; fabric; and a variety of twodimensional media. Mathematical ideas at play in the art exhibition encompassed tilings, fractals, polyhedra, hyperbolic geometry, anamorphosis, knots, topology, and magic squares.

On the threshold of the digital age, it was a great pleasure to see the acrylic, watercolor, pigment, and oil paintings of Aurora, Anita Chowdry, and János Saxon-Szász at the exhibition. The process of creation and the pure sensation of the color, just like the materiality of the medium, play a central role in their art: "I do not use a calculator in order to create these paintings; I use my mind to do the math. The value of this process is that, as the painting is completed, I embody these patterns and concepts and carry them within myself." So writes Aurora in her artist's statement. Chowdry's techniques are based on her research into the methods and materials of painting and illuminating in Indian and Persian manuscripts.

Browsing among the great variety of 3D artworks, the visitors could play with Xavier De Clippeleir's tricky transforming bodies and admire Nicholas Durnan's carved alabaster sculptures (a Möbius and two variations on the theme of Borromean rings) and Bente Simonsen's beautifully mirroring steel objects. They could also study the magical skillfulness of woodcarving by Bjarne

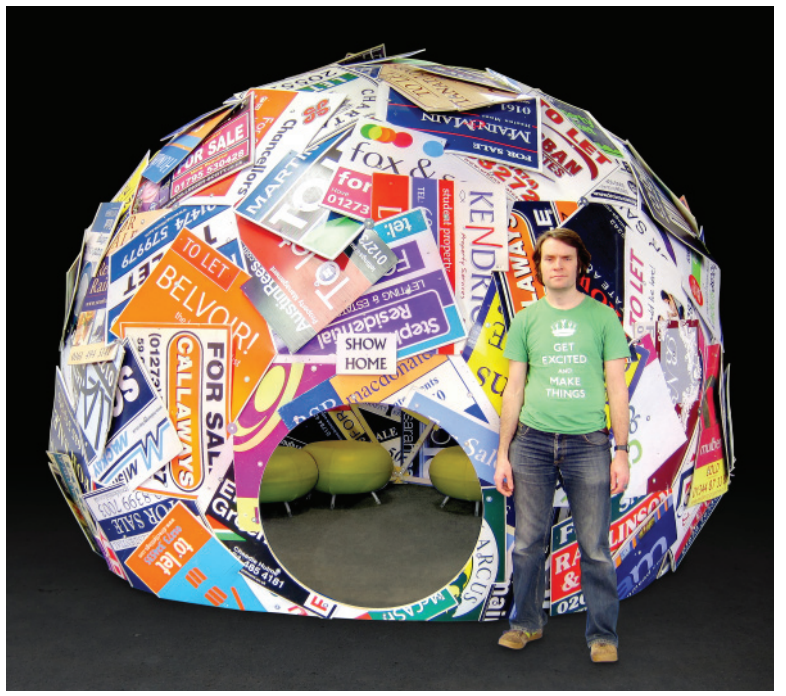

Nick Sayers: Show Home, 2011.

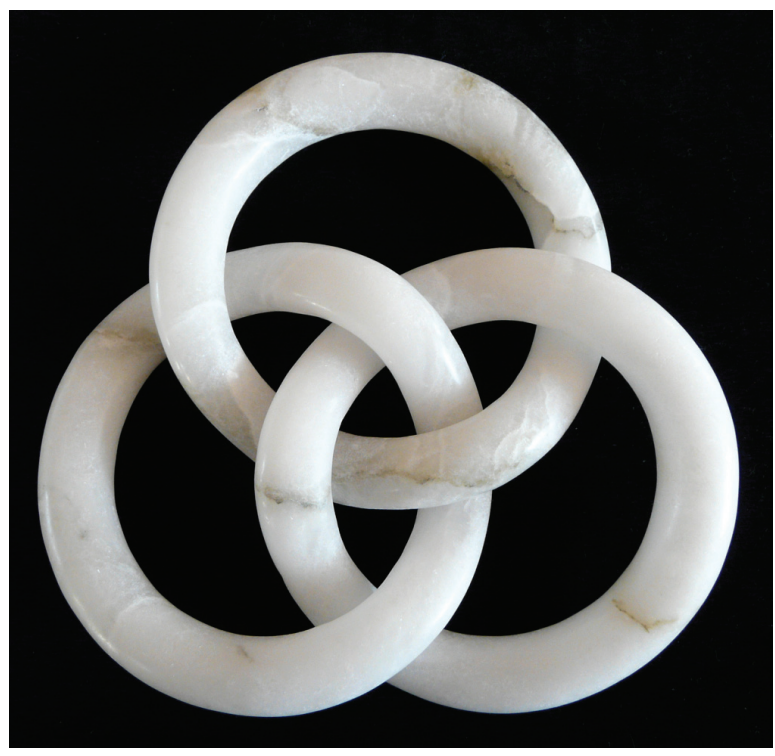

Nicholas Durnan: Borromean Rings 2, 50 x 220 x $220 \mathrm{~mm}$, English alabaster, 2011.

Jespersen. ${ }^{2}$ From the exhibited photographs of Nick Sayers's artworks, we could also get a taste of using mathematics in contemporary conceptual "bricolage-art".

Unlike much mathematical art that is purely abstract, Sayers uses recognizable household objects to create work that is accessible, real, and fun. The largest work he presented, as a photograph, at the exhibit was Show Home: a four-meter-indiameter, three-meter-high geodesic shelter built from 135 estate agent (realtor) "For Sale" and "To Let" boards. The piece made a statement about homelessness, the housing market, and sustainable architecture.

\footnotetext{
${ }^{2}$ Bjarne Jespersen, Woodcarving Magic. How to Transfer a Single Block of Wood Into Impossible Shapes, East Petersburg, PA: Fox Chapel Publishing, 2012.
} 


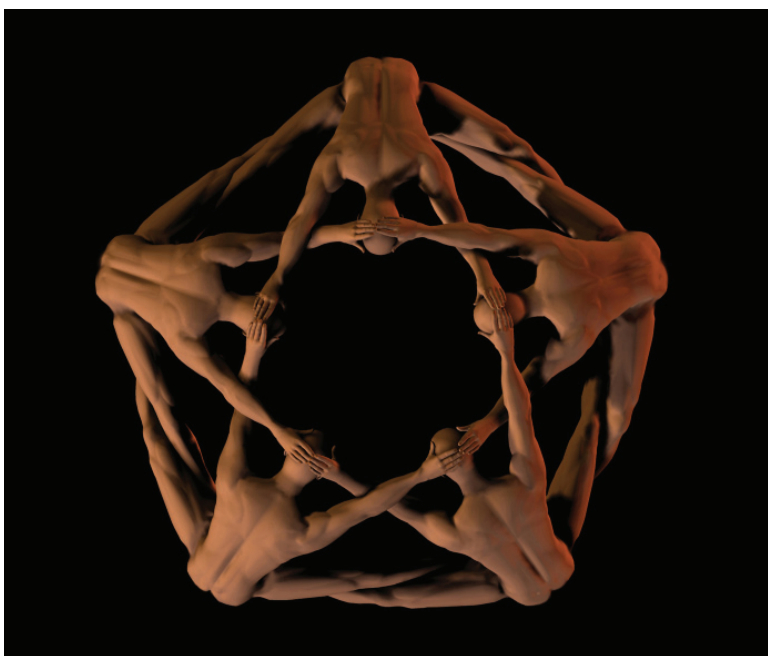

Mike Naylor: Pentamen, 16" x 12", digital print, 2002.
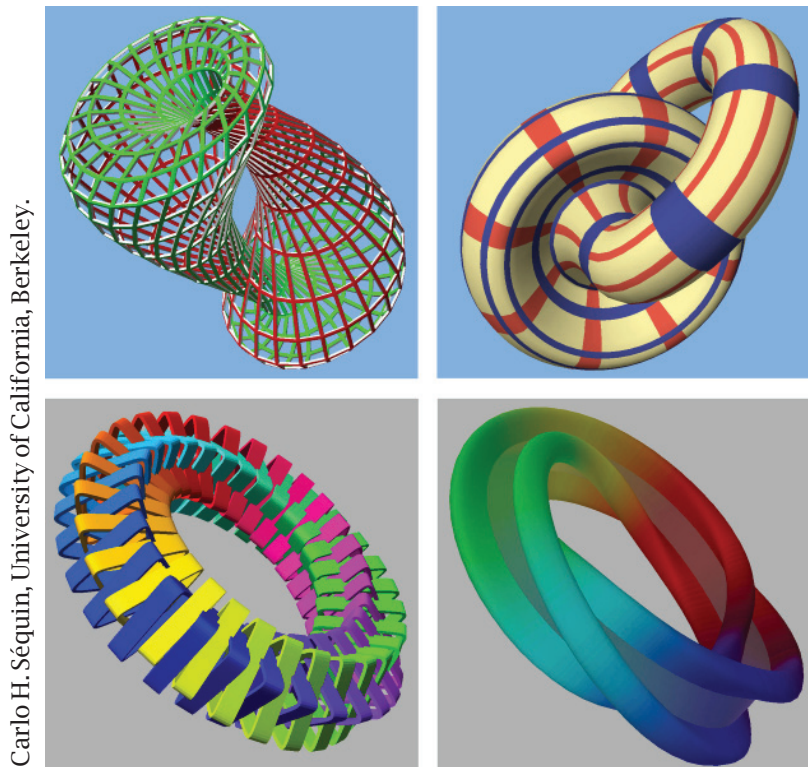

Carlo Séquin: The World of Wild and Wonderful Tori, 24" x 24", composite of computer images, 2011.

After seeing the pieces of Mike Naylor's geometric body art, the audience could be convinced about the naked truth of the artist's simple statement: "Mathematics is part of us, and we are part of mathematics." Moving away from the mathematically obsessed nude people towards the dressed ones, we could enjoy remarkable examples of mathematically inspired fashion design by Jasmin Schaitl and Eunsuk Hur. By the fusion of mathematics and fashion Schaitl's model shapes and defines the body in a new context and opens up a variety of exciting design processes. Hur's modular textile pieces also encourage the end user to participate in the design process by allowing the user freedom to personalize the design through playful experimentation.
Computer-generated graphics and animation art were also represented on a high-quality level. Mingjang Chen used the Structural Cloning Method (SCM) and the Leaping Iterated Function System (LIFS) to explore abstract and landscape paintings. Gary Greenfield's Transport Network Overlay series are based on simulations that are inspired by mathematical models of physical and biological processes. Mehrdad Garousi used Mandelbulb 3D for adventurous explorations into his extended, three-dimensional fractal worlds. Daniela Rinaudo, in a philosophical piece called Geometric Man, used computer animation to offer a virtual journey for the spectator into "imaginary time" in search of another dimension into space. Nathan Selikoff experimented with chaotic attractors. Carlo Séquin (University of California, Berkeley), a plenary speaker, who together with Reza Sarhangi, took a major role in the organization of the scientific program of the conference, exhibited his 3D artworks. He also exhibited his computer graphics to visually support his plenary talk, the "Tori-Story". His presentation elaborated on the classification of all topological tori into four regular homotopy classes, where the members in one class cannot be smoothly transformed into members of another class. His art submissions depicted some intriguing structures that topologically are torus surfaces but with enough surprising contortions so that most people would not immediately see them as your everyday donut.

We saw innovative approaches in uniting fractals and tessellation art by Robert Fathauer, through the combination of traditional art forms like photography with digital techniques to create tree-like and knotted spiral designs that have an intriguing blend of complexity and beauty.

Design works are always in high favor at the Bridges exhibits, especially when broadly celebrated designers like Fabien Vienne from France bring the honor of their presence to the exhibition. Vienne's beautiful creations convey the notion that geometry is no mere tool of composition; it's an epistemology. Each of his works seeks to employ the principle of economy to find the essential in a problem and to then "substantiate" it in a least action solution, which brings that essence to light and to life.

\section{Mathematics Is Culture}

In 2011, for the second time in the Bridges' history, the music night was organized by Dmitri Tymoczko, a composer and music theorist at Princeton University. Coimbra's professional orchestra, the Orquestra Clássica do Centro, had graciously agreed to play mathematically inspired and inspiring works, both new and old. The concert featured a newly composed piano concerto by Dmitri Tymoczko based on the idea of cellular automata, a recent piece by Giovanni Albini based on the idea 

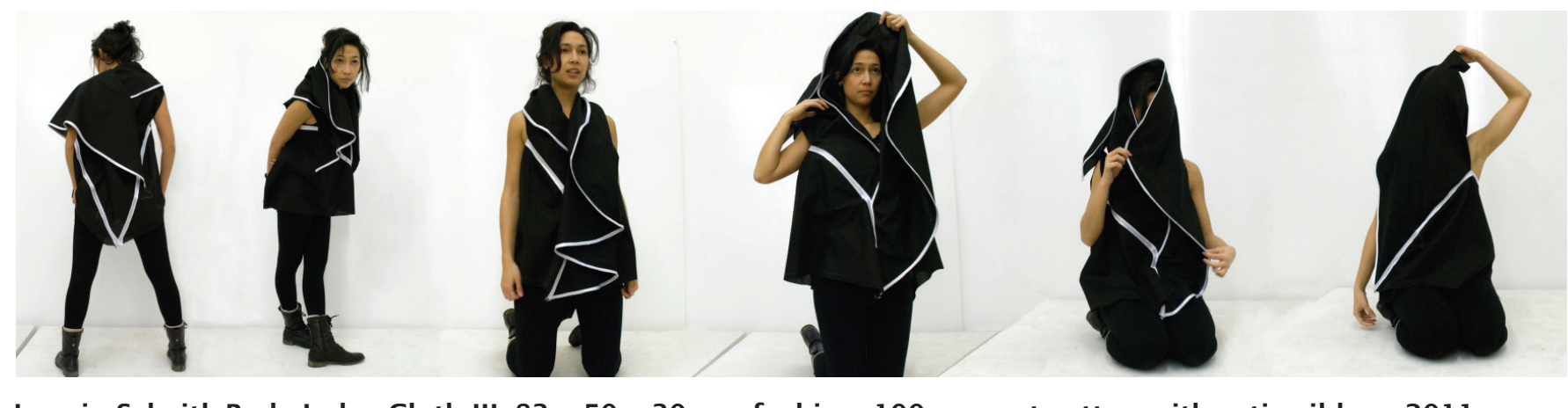

Jasmin Schaitl: Body-Index-Cloth III, 83 x 50 x 30 cm, fashion, 100 percent cotton with satin ribbon, 2011.

of symmetry, and an older piece by Tom Johnson in which a mathematics theorem is proved before one's very ears! Maestro Artur Pinho's orchestra performed magnificently.

The theater night was orchestrated by Steve Abbott (Middlebury College), a researcher of the intersections of mathematics and theater and the coeditor of Math Horizons. The performance focused on a single dramatic piece: The Physicists, by Friedrich Dürrenmatt, performed in the form of a staged reading by the conference participants themselves.

A rapidly growing number of artists and educators are using movies, videos, and animations for applications spanning education, industry, and the arts. The Bridges math/art short movie festival, which was directed by Amy Christie and Nathan Selikoff, screened many short movies, including a virtual reenactment of Escher's Drawing Hands, a demonstration of the bubble-sort algorithm via Hungarian folk dance, and an animation, the Adventures of the Klein Bottle, by Konstantin Weixelbaum and Ilkay Sakalli, supervised by Konrad Polthier (Free University, Berlin).

Poetry day was a new addition this year to the Bridges Conference. This event was coordinated by Sarah Glaz, a mathematics professor from the University of Connecticut and a poet. There was a reading of poems with strong links to mathematics. Reading their own poems were Emily Grosholz, JoAnne Growney, Amy Uyematsu, and Sarah Glaz. Saeed Ghahramani (a mathematician and the dean of arts and sciences at Western New England University) read translated modern Iranian poems. Coimbra University mathematician and translator Francisco Craveiro was joined by other poets for a bilingual reading of mathematical poems that he translated into Portuguese. Craveiro read the Portuguese translations, while the other poets read the English originals. The event ended with an "open microphone" period in which Bridges participants could share their own mathematical poems with the audience.

\section{Mathematics Is Fun}

Mathematics can be scientific, and it can be artistic as well. But can mathematics be playful? Can

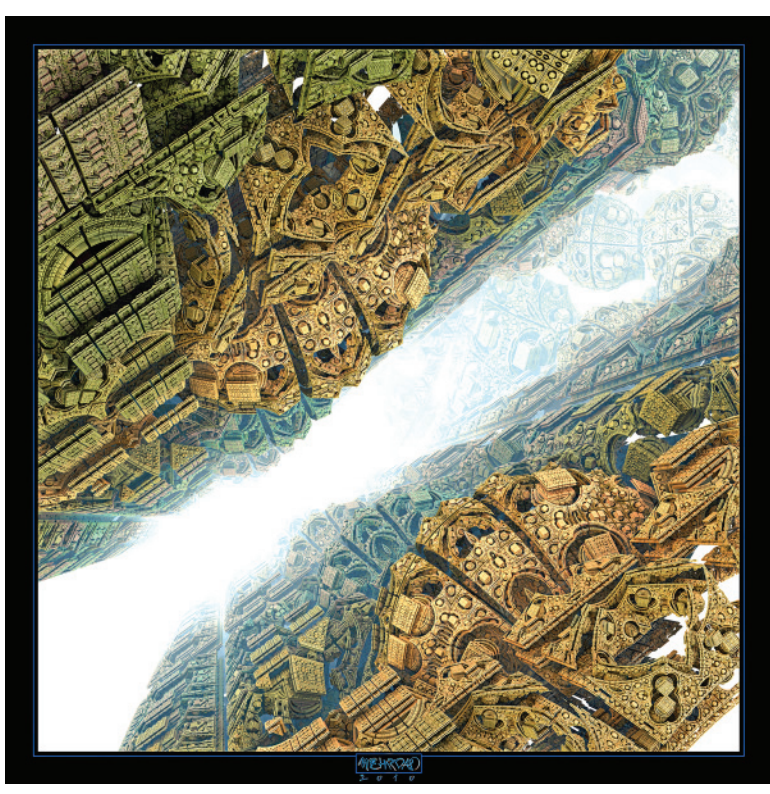

Mehrdad Garousi: Space Shelter, 20" x 20", digital print, 2010.

it be fun? We gave it a try at Bridges family day (http://www. fami1yday.hu. The participating children, parents, and all the visitors had the possibility, during the workshops, to test themselves as math-fashion designers with the leadership of the enthusiastic artist team of Ruth Mateus-Berr from the University of Applied Arts in Vienna; or as mathematical sculptors with Nicholas Durnan; or as weavers or quilters with Elaine Krayenke Ellison. We made geometry with our own bodies at the Human Geometry Workshop led by Mike Naylor and Vi Hart; enjoyed the miracle of kaleidoscopes with Curtis Palmer; practiced mathematical origami with Wojtek Burczyk; learned some tricks from a real mathe-magician, Fernando Blasco; played amazing puzzles with the internationally renowned designer Jean-Marc Castera; took a quick tour in the "Vasarely Playhouse" with Slavik Jablan; played with the giant triangles of Simon Morgan and Jacqueline Sack; worked with clay under the direction of Jouko Koskinen; immersed ourselves in the secrets of Islamic mosaic design with Sarah Abdellahi and Tom Goris; and tried the Zometool 


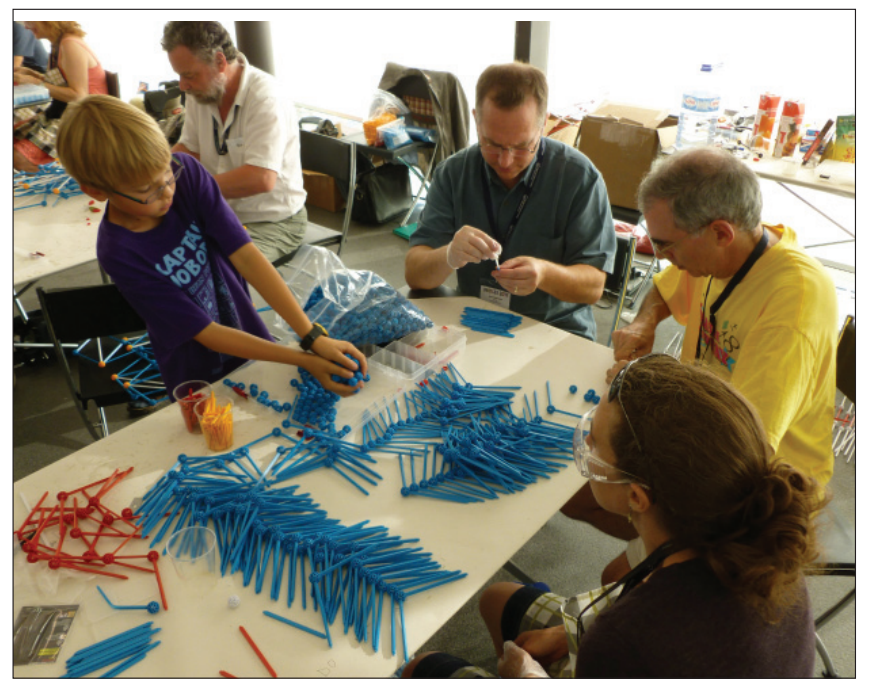

The Zometool Pentigloo construction team at work. Derek Vorthman (left), László Vörös (left, background), Scott Vorthman (middle), George W. Hart (right), Kelly Delp (right, foreground). (Photo by Samuel Verbiese.)
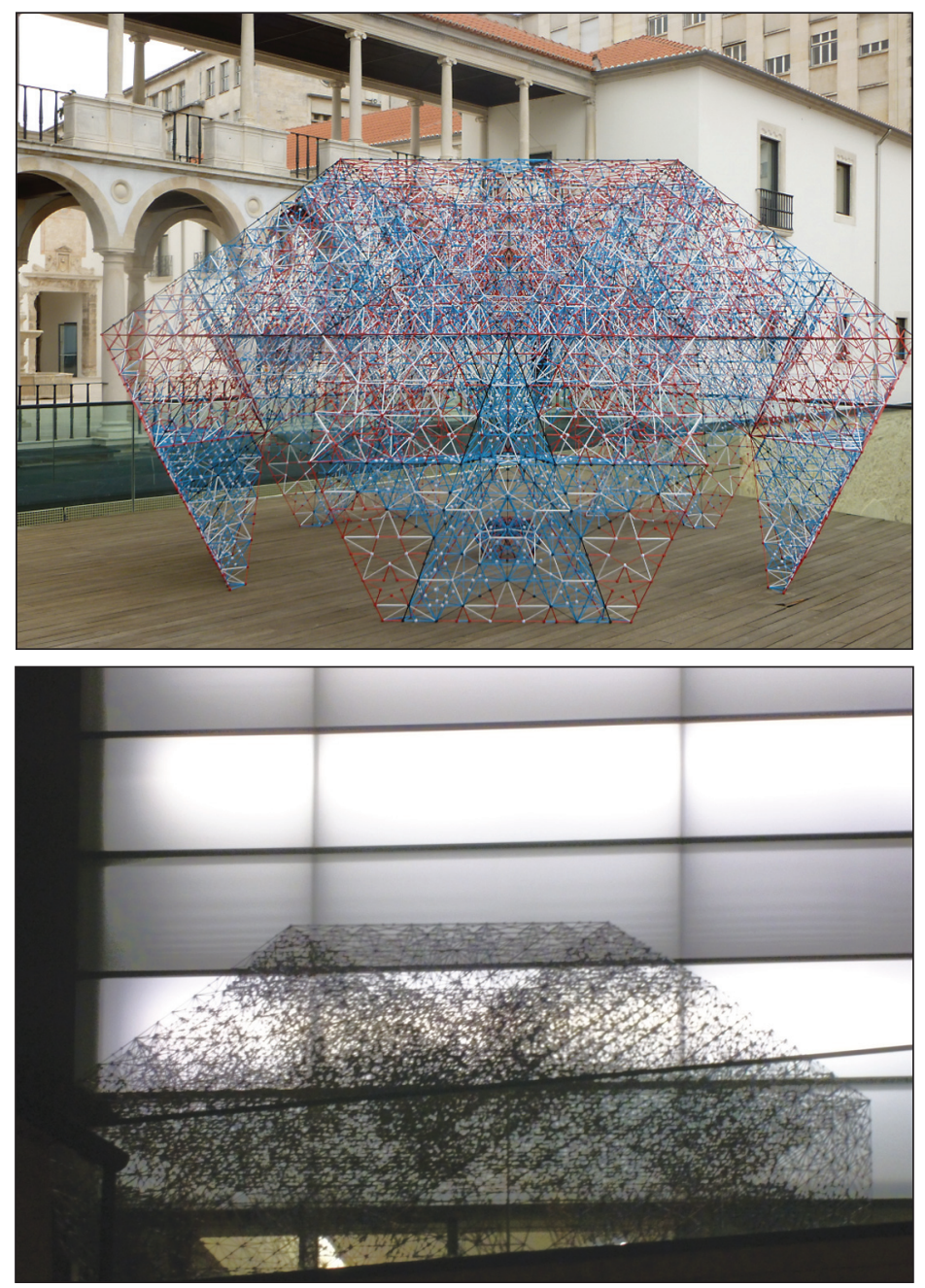

Above: Bridges around the clock: Fabien Vienne's Pentigloo at day and at night in the Museu Nacional de Machado de Castro, Coimbra. (Photos by Samuel Verbiese.) modeling kit with Paul Hildebrandt, Mike Stranahan, and Samuel Verbiese. The Zometool modeling kit was in use during the conference in the Castro Machado Museum of Coimbra to build up the ambitious Pentigloo: an incredible construction of 44,771 parts with a diameter of almost nineteen feet by Fabien Vienne, the noted 86-year-old designer who was present and worked together with the construction team (led by Paul Hildebrandt and Jim Hausman) during the construction process.

Such a rich and mosaic-like event, which brought participants from more than thirty countries in the world, could not be put together by just a few official chair persons. In addition to the 2011 Bridges Program Committee of about forty individuals and the organizers of the many events described above, I should mention the important roles of the organizing team in Coimbra, including Ana Almeida from the Department of Physics and Mathematics, ISEC-Instituto Superior de Engenharia de Coimbra; Amílcar Cardoso, the president of the Centre for Informatics and Systems, University of Coimbra, Portugal; and Penousal Machado from the Department of Computer Science, University of Coimbra, Portugal.

The next Bridges event will take place on July 25-29, 2012, at Towson University, located in the Baltimore metropolitan area, Maryland, USA. Please find more information at http://www. BridgesMathArt.org/Bridges-2012. 